\title{
Necrotizing sarcoid granulomatosis with extrapulmonary involvement
}

\author{
R.S. Dykhuizen*, C.C. Smith*, M.M. Kennedy**, K.A. McLay+, \\ J.S. Cockburn++, K.M. Kerr*
}

\begin{abstract}
Necrotizing sarcoid granulomatosis with extrapulmonary involvement. R.S. Dykhuizen, C.C. Smith, M.M. Kennedy, K.A. McLay, J.S. Cockburn, K.M. Kerr. CERS Journals Ltd 1997.

ABSTRACT: Pulmonary lesions, with sarcoid-like granulomas exhibiting noncaseous necrosis, with associated granulomatous arteritis fulfil the diagnostic criteria of necrotizing sarcoid granulomatosis (NSG). We report the case of a woman who presented with recurrent headaches, transient right hemipareses and left-sided ophthalmoplegia. An excised left retro-orbital lesion demonstrated sarcoid like changes, and the illness responded to steroid therapy.

Twelve years later, the patient developed a tumour in the right lung. The resected specimen showed the histological hallmarks of NSG, and careful review of the retro-orbital lesion, removed 12 years previously, revealed similar histology.

Extrapulmonary involvement in NSG is rare and has been histologically proven on only one previous occasion. The presentation of necrotizing sarcoid granulomatosis in two different systems 12 years apart is unusual and was considered worth reporting.
\end{abstract}

Eur Respir J., 1997; 10: 245-247.
Depts of *Infection, **Pathology, ${ }^{+}$Otolaryngology, and ++Thoracic Surgery, Royal Infirmary, Forresterhill, Aberdeen, UK.

\section{Correspondence: R.S. Dykhuizen}

Infection Unit

Aberdeen Royal Infirmary

Forresterhill

Aberdeen AB2 2ZB

UK

Keywords: necrotising sarcoid granulomatosis

Received: July 301995

Accepted after revision August 251996
Necrotizing sarcoid granulomatosis (NSG) was first described by LieBow [1] in 1973. He defined five forms of pulmonary angiitis and granulomatosis unrelated to infection or connective tissue disorders: Wegener's granulomatosis; limited Wegener's disease; lymphomatoid granulomatosis; bronchocentric granulomatosis; and NSG.

Isolated reports of patients with this disorder have appeared in the literature [2-5], describing pulmonary lesions with sarcoid-like granulomas showing varying degrees of noncaseous necrosis and granulomatous arteritis [1, 3, 4, 6, 7]. Extrapulmonary involvement is rare [3, 6-8], and the histological features of NSG outside the lungs have been reported only once previously [6].

We report the case of a woman who developed granulomatous lesions in the left orbit and the right lung 12 yrs apart. Both lesions showed the histological hallmarks of NSG.

\section{Case report}

The patient, who was born in 1944, was a mother and housewife, and had smoked 10-20 cigarettes a day all her life. She presented on several occasions between 1974 and 1976 at the Aberdeen Royal Infirmary with headaches, which were treated as migraine or tension headaches with limited success.

In March 1979, she was admitted with fever, global weakness of the right upper limb and an extensor plantar response. Computerized tomography (CT) head scan and lumbar puncture revealed no abnormalities. Erythrocyte sedimentation rate (ESR) was normal, and antinuclear factor (ANF) and rheumatoid factor (RF) were negative. The illness improved spontaneously over several days.

In December 1981, the patient was readmitted with fever, headache, photophobia and diplopia. Neurologi cal examination revealed a left 6th nerve palsy, a left lower motor neurone 7 th nerve palsy, and reappearance of the right extensor plantar response. Her ESR, ANF, RF, and CT head scan were unremarkable, but the cerebrospinal fluid (CSF) protein was raised at $900 \mathrm{mg} \cdot \mathrm{L}^{-1}$ (reference $<430 \mathrm{mg} \cdot \mathrm{L}^{-1}$ ), with normal glucose and no pleocytosis. Oligoclonal bands were absent. A vasculitic process in the region of the left middle cerebral artery was considered, and steroid therapy resulted in symptomatic and clinical improvement. Once the steroid medication was reduced, however, she developed a left ophthalmoplegia. Magnetic resonance imaging (MRI) revealed a small tumour in the left orbit near the left lateral rectus muscle. Histology of the resected lesion showed necrotizing granulomatous inflammation, with multinucleate giant cells and granulomatous vasculitis (fig. 1). Special stains for fungi and acid and alcohol fast bacilli (AAFB) and culture for tuberculosis proved negative.

Following a differential diagnosis of sarcoidosis or tuberculosis, the patient was treated with steroids, and rifampicin with ethambutol. The antituberculosis therapy was continued for 2 yrs. Attempts to taper off the steroids resulted in return of the headaches and right hemipareses, leading to repeated hospital admissions. On one such occasion in 1989, a routine chest radiograph 


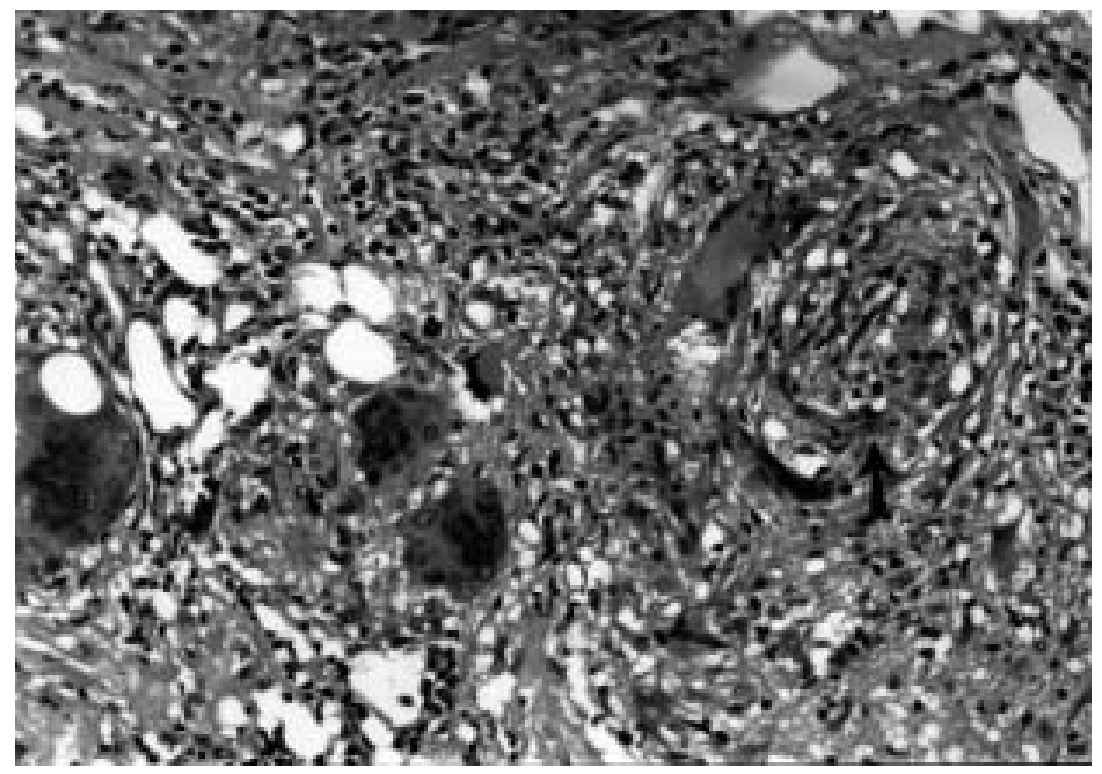

Fig. 1. - Granulomatous infiltration in the tissue from the orbit. A small vessel is involved in the granulomatous process (arrow). Scale bar $=200 \mu \mathrm{m}$.

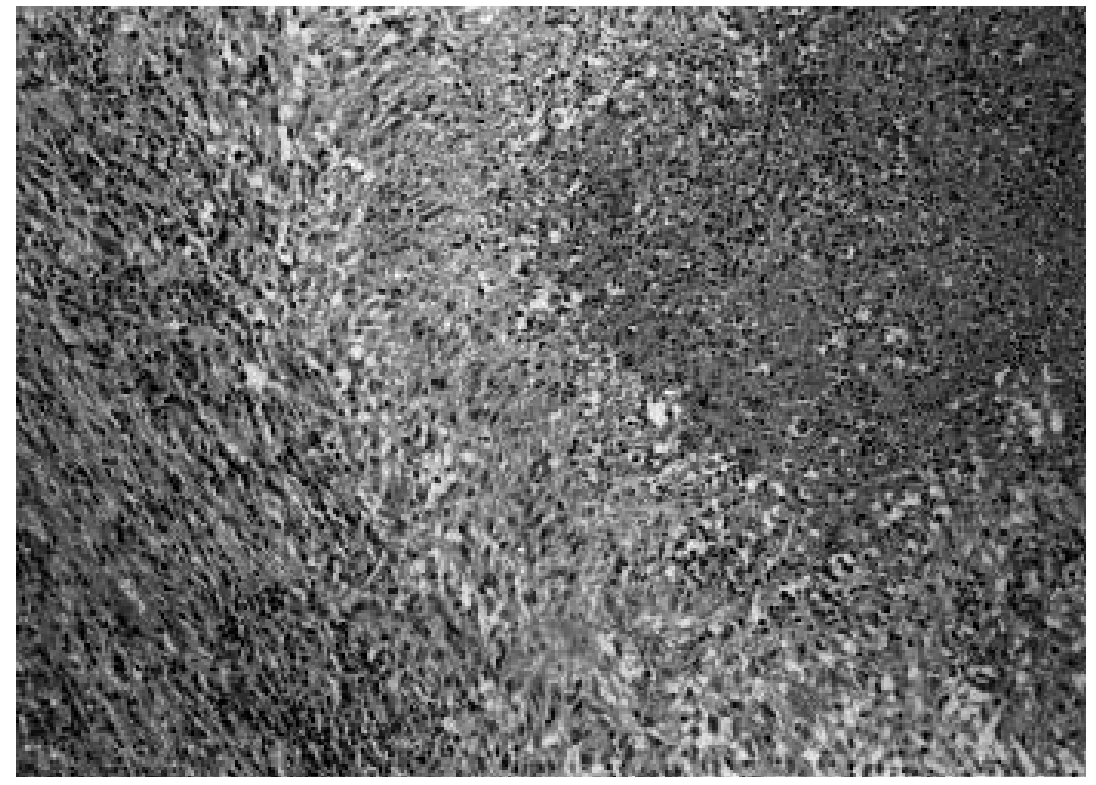

Fig. 2. - As well as showing granulomatous inflammation identical to that in figure one, the pulmonary lesion revealed parenchymal necrosis bordered by epitheloid cells, giant cells and chronic inflammation. Scale bar $=500 \mu \mathrm{m}$.

demonstrated a rounded opacity in the lower zone of the right lung and liver function tests proved abnormal: aspartate amino transferase $254 \mathrm{IU} \cdot \mathrm{L}^{-1}$ (reference interval 5-35 IU $\left.\cdot \mathrm{L}^{-1}\right)$, alkaline phosphatase $171 \mathrm{IU} \cdot \mathrm{L}^{-1}($ reference 30-300 IU $\left.\cdot \mathrm{L}^{-1}\right)$, and $\gamma$-glutamyl transferase 123 $\mathrm{IU} \cdot \mathrm{L}^{-1}$ (reference $7-33 \mathrm{IU} \cdot \mathrm{L}^{-1}$ ). The appearances of the liver on ultrasonography was normal. The opacity on the chest radiograph and the liver function abnormalities resolved with increased dexamethasone dosage.

In 1991, an indurated, tender, erythematous skin lesion appeared over the bridge of the patients nose and the adjacent left lower eyelid. CT and MRI scanning showed mucosal swelling of the maxillary and ethmoid sinuses, and mucosal biopsy revealed chronic inflammation without vasculitis or granulomatous disease. The swelling of the sinuses resolved following increased steroid dosage and antimicrobial chemotherapy.

In March 1994, a chest radiograph revealed a shadow in the right midzone, and CT scanning suggested a tumour. The right middle lobectomy specimen revealed a $4 \mathrm{~cm}$ mass of pale homogeneous material, with focal haemorrhage surrounded by endogenous lipoid pneumonia. The lymph nodes and bronchial resection margin were normal. Stains for acid-fast bacilli and fungi (and subsequent culture) proved negative. Histology of the lesion showed well-defined granulomata with giant cells, lymphocytes and plasma cells, aggregated in masses. Some were related to bronchovascular bundles. Extensive necrosis was evident within granulomata and the adjacent lung parenchyma (fig. 2). The vasculitis, composed of infiltrating lymphocytes and macrophages, was granulomatous in some areas. Acute necrotizing vasculitis was not identified.

\section{Discussion}

For some 20 yrs this patient had followed an unusual clinical course, the exacerbations being partially responsive to steroid therapy. Auto-antibodies, anticardiolipin antibody, RF, antineutrophil cytoplasmic antibody (ANCA), hepatitis $\mathrm{A}, \mathrm{B}$ and $\mathrm{C}$ serology, and serum angiotensin-converting enzyme (ACE) levels were repeatedly negative. Measurements of ESR and C-reactive protein (CRP) concentration revealed no elevation (even during exacerbations of the syndrome), and her urine was physiological throughout. Testing with Kveim antigen was never undertaken because of the continuous therapy with corticosteroids. The histology of the orbital mass was originally described as necrotizing granulomatous inflammation with multinucleate giant cells and granulomatous vasculitis, without making reference to the diagnosis of necrotizing sarcoid granulomatosis. The right middle lobe of the lung, resected 12 yrs later, was examined by a different pathologist, who identified the histological appearance of necrotizing sarcoid-like granulomatous inflammation. The biopsy material of the previous orbital mass was still available and on review showed identical histology. The long duration of the illness, its response to steroids, and the negative tissue cultures make an infectious aetiology unlikely. Wegener's granulomatosis was excluded on clinical and histopathological grounds.

The relationship between NSG and classical sarcoidosis is debatable. CHURG [7] argues that NSG resembles sarcoidosis histologically, clinically and in the pattern of 
extrapulmonary involvement. Histologically however, NSG differs from sarcoidosis in that vasculitis is prominent, necrosis marked, and hilar lymphadenopathy rare. In our patient, lymph nodes and bronchial mucosa were histologically, devoid of disease and there was no evidence of interstitial granulomatous infiltration outside the lesion.

Some of the cases with extrapulmonary disease in the form of hilar node or hepatic involvement referred to by CHURG [7], might have been classical sarcoidosis rather than NSG. The one previously reported case of NSG with extrapulmonary involvement, histologically proven [6], and other cases $[3,7,8]$ suggest a predilection for the eye and central nervous system. One patient had ocular symptoms more than 10 yrs before the onset of chest symptoms [4].

NSG may well be related to sarcoidosis, but its clinical and histopathological manifestations would seem to justify its status as a separate disease entity, whether referred to as necrotizing sarcoid granulomatosis or atypical nodular sarcoidosis. Although the illness may be considered benign and responsive to corticosteroids, severe morbidity was experienced by our patient during the protracted clinical course of her disease. At present she remains well on low-dose steroids.

\section{References}

1. Liebow AA. The J. Burns Amberson lecture: Pulmonary Angiitis and granulomatosis. Am Rev Respir Dis 1973; 108: 1-17.

2. Stephen JG, Braimbridge MV, Corrin B, Wilkinson SP, Day D, Whimster WF. Necrotizing "sarcoidal" angiitis and granulomatosis of the lung. Thorax 1976; 31: 356-360.

3. Churg A, Carrington CB, Gupta R. Necrotising sarcoid granulomatosis. Chest 1979; 76: 406-413

4. Koss MN, Hochholzer L, Feigin DS, Carancis JC, Ward PA. Necrotizing sarcoid-like granulomatosis: clinical, pathologic and immunopathologic findings. Hum Pathol 1980; 11 (Suppl.): 510-519.

5. Spiteri MA, Gledhill A, Campbell D, Clarke SW. Necrotising sarcoid granulomatosis. Br J Dis Chest 1987; 81: 70-75.

6. Singh N, Cole S, Krause PJ, Conway M, Garcia L. Necrotizing sarcoid granulomatosis with extrapulmonary involvement: clinical, pathologic, ultrastructural and immunologic features. Am Rev Respir Dis 1981; 124: 189-192.

7. Churg A. Pulmonary angiitis and granulomatosis revisited. Hum Pathol 1983; 14: 868-883.

8. Beach RC, Corrin B, Scopes JW, Graham E. Necrotizing sarcoid granulomatosis with neurological lesions in a child. J Pediatr 1980; 97: 950-953. 